\title{
Intervenções educativas na prevenção ou tratamento da obesidade em adolescentes: revisão integrativa ${ }^{1}$ \\ Interventions in the prevention or treatment of obesity in adolescents: integrative review Intervenciones educativas en la prevención o tratamiento de la obesidad en adolescentes: revisión integrativa
}

Maria Aline Moreira Ximenes ${ }^{2}$, Maria Girlane Sousa Albuquerque Brandão ${ }^{3}$, Josiane da Silva Gomes ${ }^{4}$, Francisco
Marcelo Leandro Cavalcante ${ }^{5}$ Joselany Áfio Caetano $^{6}$, Lívia Moreira Barros ${ }^{7}$

\section{RESUMO}

Objetivo: identificar a eficácia de intervenções educativas para prevenir ou tratar a obesidade em adolescentes. Método: foi uma revisão integrativa da literatura, a partir da questão norteadora: "Qual a efetividade das intervenções de prevenção / tratamento da obesidade em adolescentes?" A busca foi realizada em seis bases de dados eletrônicas nos idiomas português, inglês e espanhol, em julho de 2020, o que permitiu a identificação de 5.401 artigos. Resultados: foram identificados 31 artigos que abrangem intervenções educativas baseadas no suporte nutricional e psicológico, atividade física e estilo de vida saudável. Predominaram atividades físicas, intervenções interdisciplinares comportamentais, nutricionais e de estilo de vida. As ações tiveram um efeito benéfico na qualidade de vida dos participantes, índice de massa corporal, hábitos de exercícios diários e comportamento alimentar positivo entre adolescentes. As intervenções para controle e prevenção da obesidade analisadas nesta revisão tiveram efeitos positivos na redução do peso corporal. Conclusão: os achados apontaram para a necessidade de mais estudos para maior controle dos benefícios e adesão às intervenções voltadas para a prevenção e tratamento da obesidade.

Descritores: Adolescente; Educação Alimentar e Nutricional; Educação em Saúde; Exercício Físico; Obesidade.

\footnotetext{
${ }^{1}$ Data de recebimento: 01 de setembro de 2020

Data de aceitação: 25 de novembro de 2020

${ }^{2}$ Enfermeira. Universidade Federal do Ceará (UFC). Brasil. E-mail: aline.ximenes11@ hotmail.com ORCID: https://orcid.org/00000002-1674-3357

${ }^{3}$ Enfermeira. Universidade da Integração Internacional da Lusofonia Afro-Brasileira (UNILAB). Brasil. E-mail: girlane.albuquerque@ yahoo.com.br ORCID: https://orcid.org/0000-0002-9925-4750

${ }^{4}$ Enfermeira. Universidade Federal do Ceará (UFC). Brasil. E-mail: josianegomes4@ hotmail.com ORCID: https://orcid.org/0000-00018130-9795

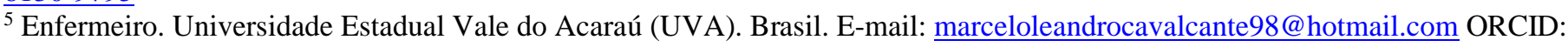
https://orcid.org/0000-0001-6143-1558

${ }^{6}$ Enfermeira. Universidade Federal do Ceará (UFC). Brasil. E-mail: joselany @ ufc.br ORCID: https://orcid.org/0000-0002-0807-056X

${ }^{7}$ Enfermeira. Universidade da Integração Internacional da Lusofonia Afro-Brasileira (UNILAB). Brasil. E-mail:

livia.moreirab@hotmail.com ORCID: https://orcid.org/0000-0002-9763-280X
} 


\section{ABSTRACT}

Objective: to identify the effectiveness of educational interventions to prevent or treat obesity in adolescents. Method: it was an integrative review of the literature, based on the guiding question: "What is the effectiveness of obesity prevention / treatment interventions in adolescents?" The search was carried out in six electronic databases in Portuguese, English and Spanish in July 2020, which allowed the identification of 5,401 articles. Results: 31 articles were identified that cover educational interventions based on nutritional and psychological support, physical activity and a healthy lifestyle. Physical activity, interdisciplinary behavioral, nutritional and lifestyle interventions predominated. The actions had a beneficial effect on the participants' quality of life, body mass index, daily exercise habits, and positive eating behavior among adolescents. The interventions for the control and prevention of obesity analyzed in this review had positive effects on reducing body weight. Conclusion: the findings pointed to the need for more studies to have greater control over the benefits and adherence to interventions aimed at preventing and treating obesity.

Descriptors: Adolescent; Food and Nutrition Education; Health Education; Obesity; Physical Exercise.

\section{RESUMEN}

Objetivo: identificar la efectividad de las intervenciones educativas para prevenir o tratar la obesidad en adolescentes. Metodo: Sse trató de una revisión integradora de la literatura, basada en la pregunta orientadora: “¿Cuál es la efectividad de las intervenciones de prevención / tratamiento de la obesidad en adolescentes?". La búsqueda se realizó en seis bases de datos electrónicas en portugués, inglés y español en julio de 2020, lo que permitió identificar 5401 artículos. Resultados: se identificaron 31 artículos que cubren intervenciones educativas basadas en el apoyo nutricional y psicológico, la actividad física y un estilo de vida saludable. Predominó la actividad física, las intervenciones interdisciplinarias conductuales, nutricionales y de estilo de vida. Las acciones tuvieron un efecto beneficioso sobre la calidad de vida de los participantes, el índice de masa corporal, los hábitos de ejercicio diario y la conducta alimentaria positiva entre los adolescentes. Las intervenciones para el control y la prevención de la obesidad analizadas en esta revisión tuvieron efectos positivos en la reducción del peso corporal. Conclusión: los hallazgos señalaron la necesidad de realizar más estudios para tener un mayor control sobre los beneficios y la adherencia a las intervenciones destinadas a prevenir y tratar la obesidad

Descriptores: Adolescente; Educación Alimentaria y Nutricional; Educación para la salud; Ejercicio Físico; Obesidad. 


\section{Revista Electrónica Enfermeria Actual en costa Rica}

\section{INTRODUÇÃO}

A obesidade apresenta-se como um problema mundial de saúde pública cada vez mais prevalente em adolescentes. É uma Doença Crônica Não-Transmissível (DCNT), evitável, caracterizada pela elevação da massa adiposa corporal, que pode resultar em inúmeros prejuízos à saúde, como doenças cardiovasculares, diabetes e alguns tipos de câncer ${ }^{1,2}$.

De acordo com Organização Mundial de Saúde (OMS), a obesidade quase que triplicou no período de 1975 a 2016 , constatando-se que mais de 340 milhões de crianças e adolescentes na faixa etária de 5 a 19 anos apresentavam índice de massa corporal (IMC) fora da curva-padrão no ano de 2016, com aumento de $18 \%$ na prevalência deste problema crônico ${ }^{1}$.

Estudos realizados nos Estados Unidos ${ }^{3}$ e Emirados Árabes ${ }^{4}$ destacam a obesidade como importante problema de saúde pública, com implicações graves para a saúde, como comorbidades relacionadas com fatores fisiológicos e psicológicos. Pesquisa realizada em escolas de 21 países europeus identificou números preocupantes de adolescentes com obesidade grave, e assim, destacou a relevância de a obesidade ser tratada por meio de uma variedade de intervenções educativas, desde a prevenção precoce do sobrepeso e da obesidade, até o tratamento daqueles que precisam ${ }^{5}$.

Intervenções educativas têm sido relatadas como estratégia potencial de fomento a mudanças de estilo de vida de adolescentes, acompanhada de melhoria do perfil antropométrico e de composição corporal. Protocolo de intervenção com teor educativo pode oferecer metodologia de fácil adaptação e baixo custo para serviços de saúde, com alta adesão e baixa taxa de abandono ${ }^{6}$.

Estudos consultados inferem que intervenções educativas podem contribuir significavamente para redução IMC, massa gorda relativa e absoluta, melhora na autoestima e imagem corporal em relação à percepção do estado físico e diminuição de medo ${ }^{7,8}$.

Assim, o desenvolvimento de intervenções e programas educativos voltados para promoção de comportamentos de saúde positivos podem favorecer a redução e controle da incidência de sobrepeso e obesidade. Ressalta-se a importância da orientação de profissionais de saúde em relação aos hábitos saudáveis que podem ter impacto significativo na redução dos agravos à saúde decorrentes da obesidade e melhorar a qualidade de vida dos adolescentes ${ }^{9,10}$.

Deste modo, a identificação da efetividade de programas e intervenções educativas na prevenção ou tratamento da obesidade, disponíveis na literatura científica, pode contribuir para a prática profissional ao disponibilizar evidências sobre estratégias eficazes e adequadas ao público-alvo. Além disso, permitirá a identificação de lacunas do conhecimento e suscitar novas práticas de prevenção e tratamento para esta problemática.

Ante o exposto, este estudo objetivou identificar a efetividade de intervenções educativas na prevenção ou tratamento da obesidade em adolescentes. 


\section{Revista Electrónica Enfermeria Actual en costa Rica}

\section{MÉTODO}

Trata-se de revisão integrativa da literatura, método de pesquisa que permite a análise da literatura de forma sistemática, além de divulgar dados científicos produzidos por outros autores ${ }^{11}$. O corpus do estudo foi formado por produções científicas que evidenciavam intervenções para prevenção da obesidade em adolescentes por meio de seis etapas metodológicas ${ }^{12}$.

A questão norteadora foi construída com base na estratégia População Interesse Contexto (PICo): Qual a efetividade de intervenções sobre prevenção/tratamento da obesidade em adolescentes? Considerou-se $\mathrm{P}=$ adolescentes; $\mathrm{I}=$ intervenções de prevenção ou tratamento contra obesidade; e Co = redução/prevenção da obesidade ${ }^{13}$.

Os critérios de inclusão delimitados foram estudos nos idiomas português, inglês e espanhol, que respondessem à questão norteadora e estivessem disponíveis na íntegra eletronicamente, no período de 2000 a 2020 . Foram excluídas publicações repetidas, revisões de literatura, editoriais, manuais, dissertações e teses.

A busca dos estudos foi realizada durante o mês de julho de 2020. Esse processo ocorreu em bases de dados relevantes e de impacto para o contexto da saúde, sendo elas: Medical Literature Analysis and Retrieval Sistem Online (MEDLINE), Scientific Electronic Library Online (SciELO), Cochrane, Science Direct, Base de Dados de Enfermagem (BDENF) e Literatura Latino-Americana e do Caribe em Ciências da Saúde (LILACS).

Para a realização das buscas nas bases de dados, foram utilizados descritores controlados presentes no Medical Subject Headings (MeSH), os quais: obesity e adolescente, que foram associados com palavra-chave "intervention", formando uma estratégia de busca ampla: (Obesity AND Adolescent AND Intervention).

Após a exclusão de duplicatas por meio do gerenciador de referências do Mendeley, o processo de seleção dos estudos foi realizado de forma independente por dois revisores. Destaca-se que quando havia discordância sobre o julgamento da elegibilidade, bastava apenas um revisor julgar o artigo elegível que ele passava para a etapa seguinte.

A seleção dos estudos ocorreu em duas etapas. Inicialmente, os estudos potencialmente elegíveis foram préselecionados por meio da avaliação dos títulos e resumos das publicações recuperadas nas buscas. Na segunda etapa, foi realizada avaliação do texto na íntegra para confirmação da elegibilidade. A extração dos dados das publicações incluídas também foi realizada de forma independente entre os pesquisadores e as informações foram confrontadas. Para extrair as variáveis necessárias para atingir o objetivo proposto, utilizou-se formulário próprio de coleta de dados, elaborado pelos autores, com as seguintes variáveis: autores, ano, país do estudo, tipo de estudo, nível de evidência, amostra, ambiente, idade, nível de obesidade, tempo de seguimento, tipo de intervenção e seus elementos educativos e efetividade.

A análise dos dados da revisão integrativa foi elaborada na forma descritiva. Cada estudo incluído foi analisado minuciosamente pelos autores e elaborou-se dois quadros síntese contendo as seguintes informações: autores, ano de publicação, objetivo, cepas causadoras das epidemias/pandemia, cidade e ano de ocorrência, dimensões e construtos relacionados com a Síndrome de Burnout.

O nível de evidência definido seguiu a classificação: nível I - metanálise de estudos controlados e randomizados; nível II - estudo experimental; nível III - estudo quase experimental; nível IV - estudo descritivo/não experimental 
ou com abordagem qualitativa; nível V - relato de caso ou experiência; nível VI - consenso e opinião de especialistas $^{14}$.

\section{RESULTADOS}

Foram selecionados 31 estudos, com base nos critérios de inclusão estabelecidos. Os dados compilados contemplaram a caracterização dos estudos e as intervenções descritas em cada artigo sobre prevenção ou tratamento da obesidade em adolescentes. O fluxograma do processo de seleção está apresentado na Figura 1.

Figura 1. Fluxo de seleção dos artigos. Sobral (CE), Brasil, 2020.

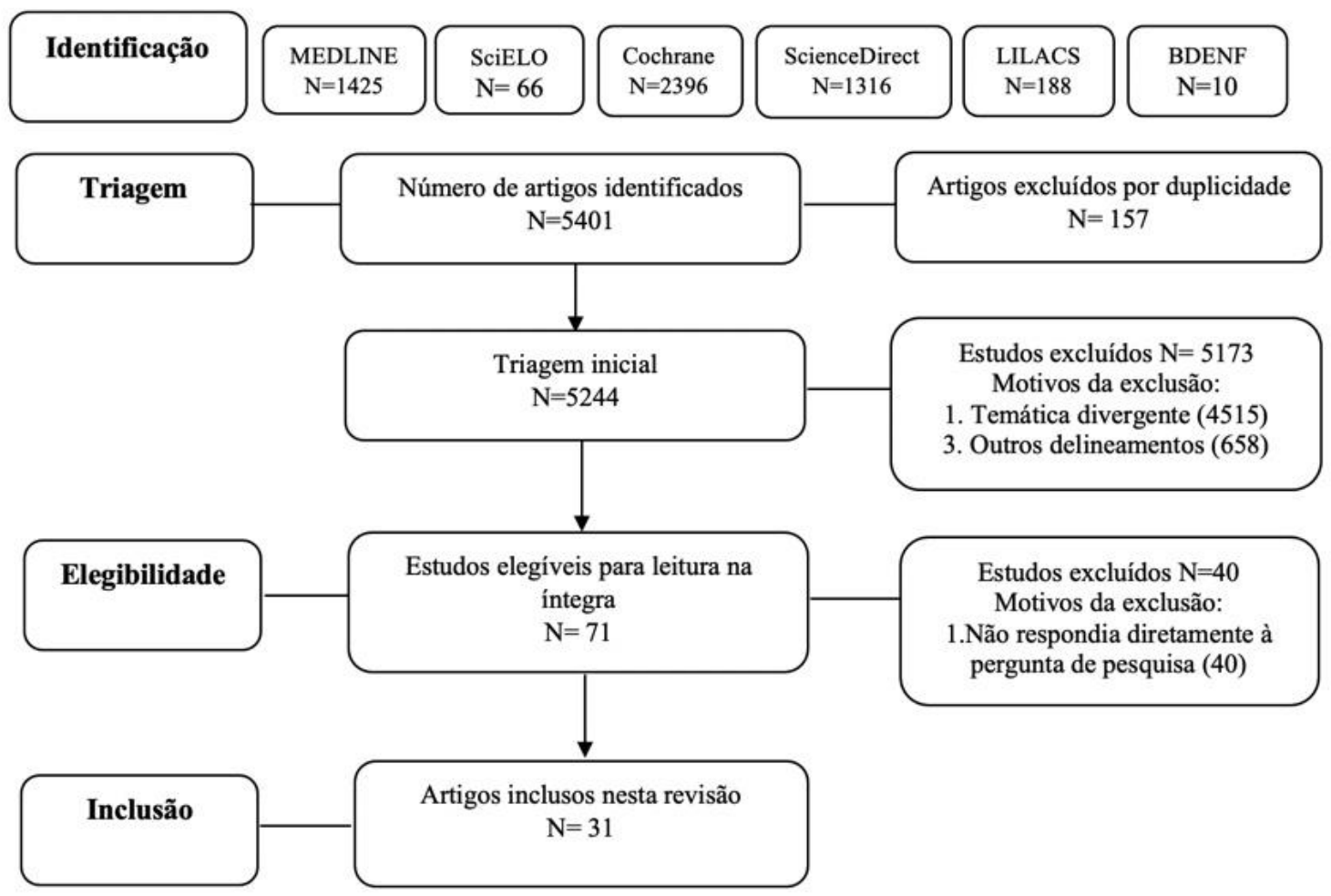

\section{Característica da amostra}

Foram selecionadas 31 publicações, provenientes de estudos realizados em países diversos, com predomínio do cenário brasileiro, efetivados majoritariamente em ambiente familiar e escolar, com adolescentes.

A maioria dos estudos usou como critérios de inclusão a avaliação do IMC, que variou de 19,94 a 36,6. Os programas de intervenção tiveram acompanhamento longitudinal, com duração de no mínimo dois e no máximo 36 meses. Estas informações estão exemplificadas no Quadro 1. 


\section{Revista Electrónica Enfermeria Actual en costa Rica}

Quadro 1 - Distribuição das características metodológicas dos artigos incluídos na revisão integrativa. Sobral (CE), Brasil, 2020.

\begin{tabular}{|c|c|c|c|c|c|c|}
\hline Autor(es)/ Ano/ País & $\begin{array}{l}\text { Tipo de estudo/ } \\
\text { Nível de } \\
\text { evidência }\end{array}$ & Amostra & Ambiente & Idade & $\begin{array}{c}\text { Nível de } \\
\text { obesidade } \\
\text { (valores de } \\
\text { IMC) }\end{array}$ & $\begin{array}{l}\text { Tempo de } \\
\text { seguimento }\end{array}$ \\
\hline $\begin{array}{l}\text { Zanatta et al., } \\
2020^{14} / \text { Brasil }\end{array}$ & Experimental/ II & 37 & $\begin{array}{l}\text { Instituição } \\
\text { de Saúde }\end{array}$ & $\begin{array}{c}15 \text { a } 18 \\
\text { anos }\end{array}$ & $\begin{array}{c}\geq \text { percentil } \\
85\end{array}$ & 3 meses \\
\hline $\begin{array}{l}\text { Ishak et al., } \\
2020^{16} / \text { Malásia }\end{array}$ & $\begin{array}{c}\text { Quase } \\
\text { experimental/III }\end{array}$ & 76 & Escolar & $\begin{array}{c}13 \text { a } 14 \\
\text { anos }\end{array}$ & $\begin{array}{l}\text { GI }-20,94 \pm \\
4,72 \\
\text { GC- } 19,94 \pm \\
5,62\end{array}$ & 3 meses \\
\hline $\begin{array}{c}\text { Chew et al.,201917/ } \\
\text { Singapura }\end{array}$ & Experimental/II & 61 & Familiar & $\begin{array}{c}10 \text { a } 16 \\
\text { anos }\end{array}$ & $\begin{array}{c}\text { GI }-29.93 \pm \\
4.24 \\
\text { GC- } 30.11 \\
\pm 4.43\end{array}$ & 6 meses \\
\hline $\begin{array}{l}\text { Poll et al., } \\
\text { 2019'8/Brasil }\end{array}$ & Experimental/II & 62 & Escolar & $\begin{array}{c}\text { Média } \\
\text { GI - } 13.2 \pm \\
1.5 \\
\text { GC- } 13.0 \pm \\
1.8\end{array}$ & $\begin{array}{c}\text { GI }-29.3 \pm \\
6.7 \\
\text { GC }-27.4 \pm \\
4.5\end{array}$ & 6 meses \\
\hline $\begin{array}{l}\text { Branco et al., } \\
2019^{19} / \text { Brasil }\end{array}$ & Experimental/II & 33 & Familiar & $\begin{array}{c}13 \text { a } 17 \\
\text { anos }\end{array}$ & $\begin{array}{c}\text { G1- } 34,1 \pm \\
7,1 \\
\text { G2- } 33,6 \pm \\
6,2\end{array}$ & 3 meses \\
\hline $\begin{array}{c}\text { Martín-García et al., } \\
2019^{20} / \\
\text { Espanha }\end{array}$ & Experimental/II & 47 & Escolar & 7 a 16 anos & $\begin{array}{c}\text { GI- } 28,1 \pm \\
3,6 \\
\text { GC- } 28,7 \pm \\
3,2\end{array}$ & 3 meses \\
\hline $\begin{array}{l}\text { Rubin et al., 201921/ } \\
\text { Estados Unidos }\end{array}$ & $\begin{array}{c}\text { Quase } \\
\text { experimental/III }\end{array}$ & 111 & Familiar & 8 a 16 anos & $\begin{array}{c}\text { Percentis de } \\
97,3 \pm 0,3\end{array}$ & 5,5 meses \\
\hline $\begin{array}{c}\text { Costa et al., 20197/ } \\
\text { Brasil }\end{array}$ & $\begin{array}{c}\text { Quase } \\
\text { experimental/III }\end{array}$ & 17 & Familiar & $16 \pm 1$ & Indisponível & 3 meses \\
\hline $\begin{array}{l}\text { Ojeda-Rodríguez et } \\
\text { al.,2018 }\end{array}$ & Experimental/II & 107 & Familiar & $\begin{array}{c}\text { Média } \\
\text { GI-11,49 } \pm \\
2,5 \\
\text { GC-10,7 } \\
2,4\end{array}$ & $\begin{array}{c}\text { GI- } 28,52 \pm \\
4,6 \\
\text { GC- } 28,1 \pm \\
4,5\end{array}$ & 22 meses \\
\hline $\begin{array}{c}\text { Freitas et } \\
\text { al.,2017 } / \text { Brasil }\end{array}$ & Experimental/II & 76 & Familiar & $\begin{array}{c}13 \text { a } 18 \\
\text { anos }\end{array}$ & $\begin{array}{c}\text { GI- } 34,48 \pm \\
3,88\end{array}$ & 3 meses \\
\hline
\end{tabular}




\begin{tabular}{|c|c|c|c|c|c|c|}
\hline & & & & & $\begin{array}{l}\text { GC- } 34,55 \\
\quad \pm 3,36\end{array}$ & \\
\hline $\begin{array}{l}\text { Bianchini et al., } \\
2016^{8} / \text { Brasil }\end{array}$ & Experimental/II & 163 & Familiar & $\begin{array}{l}\text { Faixa etária } \\
\text { ou média } \\
\text { indisponível }\end{array}$ & $\begin{array}{c}\text { GI- } 29,2 \pm \\
7,3 \\
\text { GC- } 27,8 \pm \\
7,7\end{array}$ & 3,6 meses \\
\hline $\begin{array}{c}\text { Duman et } \\
\text { al.,2016 }\end{array}$ & Observacional/IV & 50 & Familiar & $\begin{array}{c}\text { Média } \\
12,16 \pm \\
0,99\end{array}$ & Indisponível & 2 meses \\
\hline $\begin{array}{l}\text { Tortosa-Martínez et } \\
\text { al., 2016 }\end{array}$ & $\begin{array}{c}\text { Quase } \\
\text { experimental/ III }\end{array}$ & 38 & Escolar & $\begin{array}{c}\text { Média } \\
\text { GI-12,95 } \pm \\
0,9 \\
\text { GC- } 13,1 \pm \\
0,9\end{array}$ & $\begin{array}{c}\text { GI- } 26,5 \pm \\
3,4 \\
\text { GC- } 27,2 \pm \\
3,4\end{array}$ & 5,5 meses \\
\hline $\begin{array}{l}\text { Ricci-Vitor et al, } \\
2016^{25} / \text { Brasil }^{2}\end{array}$ & $\begin{array}{c}\text { Quase } \\
\text { experimental/ III }\end{array}$ & 15 & Indisponível & $\begin{array}{c}10,93 \pm \\
2,28\end{array}$ & $28,9 \pm 8,3$ & 3 meses \\
\hline $\begin{array}{c}\text { Parra-Medina et } \\
\text { al.,2015 } 26 / \text { Estados } \\
\text { Unidos }\end{array}$ & $\begin{array}{c}\text { Quase } \\
\text { experimental/ III }\end{array}$ & 348 & Familiar & $\begin{array}{c}\text { Mediana } \\
\text { Crianças } 12 \\
(10-14)\end{array}$ & $\begin{array}{c}\text { IMC } \\
\text { Mediano } \\
34,9\end{array}$ & 2,7 meses \\
\hline $\begin{array}{l}\text { Sousa et al., } \\
2015^{27} / \text { Brasil }\end{array}$ & Observacional/IV & 48 & $\begin{array}{l}\text { Instituição } \\
\text { de Saúde }\end{array}$ & $\begin{array}{c}12 \text { a } 18 \\
\text { anos }\end{array}$ & $\begin{array}{c}\text { Média do } \\
\text { escore de Z } \\
\text { do IMC - } \\
2,661\end{array}$ & 5,5 meses \\
\hline $\begin{array}{l}\text { Fidelix et al., } \\
2015^{28} / \text { Brasil }\end{array}$ & $\begin{array}{l}\text { Quase- } \\
\text { experimental/ } \\
\text { Nível III }\end{array}$ & 183 & $\begin{array}{l}\text { Laboratório } \\
\text { de pesquisa }\end{array}$ & $\begin{array}{c}12 \text { a } 18 \\
\text { anos }\end{array}$ & $\begin{array}{l}>\text { percentil } \\
95\end{array}$ & 3 meses \\
\hline $\begin{array}{c}\text { Ryder et } \\
\text { al.,201429/Estados } \\
\text { Unidos }\end{array}$ & $\begin{array}{l}\text { Quase- } \\
\text { experimental/ } \\
\text { Nível III }\end{array}$ & 15 & Escolar & $15,0 \pm 1,0$ & $\begin{array}{l}\text { Percentil } \\
96,3 \pm 1,1\end{array}$ & 3 meses \\
\hline $\begin{array}{c}\text { Sousa et al., } \\
2014^{30} / \text { Brasil }\end{array}$ & $\begin{array}{l}\text { Experimental } \\
\text { Nível II }\end{array}$ & 989 & Escolar & $\begin{array}{c}15 \text { a } 24 \\
\text { anos }\end{array}$ & 34,5 a 34,8 & 10 meses \\
\hline $\begin{array}{c}\text { Giel et } \\
\text { al.,2013¹/Alemanha }\end{array}$ & $\begin{array}{l}\text { Quase- } \\
\text { experimental/ } \\
\text { Nível III }\end{array}$ & 41 & $\begin{array}{l}\text { Instituição } \\
\text { de Saúde }\end{array}$ & $13,7 \pm 1,4$ & $\begin{array}{l}>\text { Percentil } \\
99,5 \text { ou } \\
\text { percentil } 90\end{array}$ & 6 meses \\
\hline $\begin{array}{c}\text { Antunes et } \\
\text { al.,201332/Brasil }\end{array}$ & $\begin{array}{c}\text { Quase- } \\
\text { experimental/ } \\
\text { Nível III }\end{array}$ & 34 & $\begin{array}{l}\text { Laboratório } \\
\text { de pesquisa }\end{array}$ & $\begin{array}{c}12 \text { a } 15 \\
\text { anos }\end{array}$ & $\begin{array}{c}\text { Antes da } \\
\text { intervenção } \\
-29,5 \pm 5,1 \\
\text { Após } \\
\text { intervenção } \\
-28,9 \pm 3,4\end{array}$ & 5 meses \\
\hline
\end{tabular}




\section{Revista Electrónica Enfermeria Actual en costa Rica}

\section{www.revenf.ucr.ac.cr}

\begin{tabular}{|c|c|c|c|c|c|c|}
\hline $\begin{array}{l}\text { Milano et al., } \\
2013^{33} / \text { Brasil }\end{array}$ & $\begin{array}{l}\text { Observacional/ } \\
\text { Nível IV }\end{array}$ & 24 & Indisponível & $\begin{array}{c}10 \text { a } 16 \\
\text { anos }\end{array}$ & $\begin{array}{c}29,25 \pm \\
4,67\end{array}$ & 3 meses \\
\hline $\begin{array}{l}\text { Hintze;Cattai;Junior, } \\
2012^{34} / \text { Brasil }\end{array}$ & $\begin{array}{l}\text { Quase - } \\
\text { experimental/ } \\
\text { Nível III }\end{array}$ & 33 & $\begin{array}{l}\text { Laboratório } \\
\text { de pesquisa }\end{array}$ & $\begin{array}{c}\text { Homens- } \\
12.91 \pm 2.39 \\
\text { Mulheres- } \\
13.25 \pm 1.58\end{array}$ & $\begin{array}{l}\text { Homens- } \\
31,96 \pm 3,19 \\
\text { Mulheres- } \\
31,02 \pm 6,91\end{array}$ & 8 meses \\
\hline $\begin{array}{l}\text { Denielsson et } \\
\text { al.,2012 } \\
\text { 35/Suécia }\end{array}$ & $\begin{array}{l}\text { Observacional/ } \\
\text { Nível IV }\end{array}$ & 643 & $\begin{array}{l}\text { Instituição } \\
\text { de Saúde }\end{array}$ & 6 a 16 anos & $\begin{array}{c}\text { Média do } \\
\text { escore de } Z \\
\text { do IMC } 1,6 \\
a<3,5\end{array}$ & 36 meses \\
\hline $\begin{array}{c}\text { Silva et } \\
\text { al.,201236/Brasil }\end{array}$ & $\begin{array}{l}\text { Quase- } \\
\text { experimental/ } \\
\text { Nível III }\end{array}$ & 19 & Indisponível & $\begin{array}{c}10 \text { a } 18 \\
\text { anos }\end{array}$ & $\begin{array}{c}\text { Antes da } \\
\text { intervenção } \\
-30,44 \pm \\
4,81 \\
\text { Após } \\
\text { intervenção } \\
-29,58 \pm \\
4,82\end{array}$ & 8 meses \\
\hline $\begin{array}{c}\text { Johnston, Massey; } \\
\text { Marker-Hoffman, } \\
\text { 2012 } \\
\text { Unidostados } \\
\end{array}$ & $\begin{array}{l}\text { Quase- } \\
\text { experimental/ } \\
\text { Nível III }\end{array}$ & 115 & $\begin{array}{l}\text { Laboratório } \\
\text { de pesquisa }\end{array}$ & $18,4 \pm 0,6$ & $\begin{array}{c}23,21 \pm \\
3,28\end{array}$ & $\begin{array}{c}2 \text { meses } 1 \\
\text { semana }\end{array}$ \\
\hline $\begin{array}{c}\text { Vargas et } \\
\text { al.,201138/Brasil }\end{array}$ & $\begin{array}{l}\text { Experimental/ } \\
\text { Nível II }\end{array}$ & 331 & Escolar & $\begin{array}{c}11 \text { a } 17 \\
\text { anos }\end{array}$ & $\begin{array}{c}\text { GI- } \\
19,5 \pm 3,36 \\
\text { GC- } \\
18,9 \pm 3,18\end{array}$ & 4 meses \\
\hline $\begin{array}{c}\text { Martelo, } \\
\text { 2009 }\end{array}$ & $\begin{array}{l}\text { Experimental / } \\
\text { Nível II }\end{array}$ & 82 & Escolar & $\begin{array}{c}10 \text { e } 17 \\
\text { anos }\end{array}$ & $\begin{array}{l}>\text { percentil } \\
85\end{array}$ & 3 meses \\
\hline $\begin{array}{l}\text { Farias et al., } \\
2009^{40} / \text { Brasil }\end{array}$ & $\begin{array}{l}\text { Quase - } \\
\text { experimental/ } \\
\text { Nível IV }\end{array}$ & 383 & Escolar & $\begin{array}{c}10 \text { a } 15 \\
\text { anos }\end{array}$ & IMC $>19,0$ & 12 meses \\
\hline $\begin{array}{c}\text { Rodrigues; Boog, } \\
2006^{41} / \text { Brasil }\end{array}$ & $\begin{array}{l}\text { Observacional/ } \\
\text { Nível IV }\end{array}$ & 22 & $\begin{array}{l}\text { Laboratório } \\
\text { de pesquisa }\end{array}$ & $\begin{array}{c}11 \text { a } 16 \\
\text { anos }\end{array}$ & Indisponível & 8 meses \\
\hline $\begin{array}{c}\text { Fernandez et } \\
\text { al.,2004 } / \text { Brasil }\end{array}$ & $\begin{array}{l}\text { Experimental / } \\
\text { Nível II }\end{array}$ & 28 & Indisponível & $\begin{array}{c}15 \text { e } 19 \\
\text { anos }\end{array}$ & $\begin{array}{l}\geq \text { percentil } \\
\quad 95\end{array}$ & 3 meses \\
\hline
\end{tabular}

Fonte: Elaborado pelos autores.

\section{Métodos de intervenção}

Os estudos selecionados abrangeram variados elementos de intervenção para educação em saúde, com predomínio de orientações baseadas no apoio nutricional ${ }^{7,8,18,28,34,35,36,38}$ e prática de atividade física ou exercício físico ${ }^{7,15,29,34,35}$. 


\section{Revista Electrónica Enfermeria Actual en costa Rica}

Os estudos apontaram que é importante que as intervenções atendam às necessidades e interesses da populaçãoalvo; e que sejam iniciadas precocemente e mantidas por período de tempo adequado.

\section{Efetividade das intervenções}

Observou-se efetividade das intervenções educativas em 29 estudos. Os principais desfechos evidenciados relacionaram-se com aumento na prática de exercícios físicos ${ }^{20,21,23,24}$ melhor conhecimento ou comportamento alimentar $^{16,38,41}$ e redução de medidas de peso e outras variáveis relacionadas com o IMC ${ }^{8,27,28,34,36}$.

Quadro 2. Descrição dos elementos das intervenções educativas e efetividade das intervenções. Sobral (CE), 2020.

\begin{tabular}{|c|c|c|}
\hline Autores & Elementos da intervenção & Efetividade da Intervenção \\
\hline Zanatta et al., $2020^{14}$ & $\begin{array}{l}\text { - Ações educativas em saúde } \\
\text { - Autonomia e do empoderamento para mudança do } \\
\text { comportamento alimentar } \\
\text { - Prática de atividade física }\end{array}$ & $\begin{array}{l}\text { A intervenção de abordagem motivacional } \\
\text { interdisciplinar não impactou na capacidade de } \\
\text { exercício e níveis de atividade física habitual em } \\
\text { adolescentes com sobrepeso e obesidade. }\end{array}$ \\
\hline Costa et al., $2019^{21}$ & $\begin{array}{l}\text { - Terapia cognitivo-comportamental semanal } \\
\text { - Reeducação alimentar duas vezes por semana } \\
\text { - Prática de atividades físicas três vezes por semana }\end{array}$ & $\begin{array}{l}\text { As intervenções interdisciplinares promoveram } \\
\text { melhora na imagem corporal em relação à } \\
\text { percepção do estado físico e diminuição de medo. } \\
\text { Houve ligeiras mudanças no comportamento dos } \\
\text { adolescentes. }\end{array}$ \\
\hline $\begin{array}{l}\text { Bianchini et al., } \\
2016^{24}\end{array}$ & $\begin{array}{l}\text { - Intervenção psicológica semanal por uma hora } \\
\text { - Intervenção nutricional semanal por uma hora } \\
\text { - Intervenção semanal por profissionais da Educação } \\
\text { Física } \\
\text { - Consultas individuais de pais e adolescentes com } \\
\text { pediatra } \\
\text { - Intervenção psicológica com pais ou responsáveis }\end{array}$ & $\begin{array}{l}\text { Após a intervenção houve melhorias } \\
\text { significativas nas variáveis IMC, circunferência } \\
\text { de cintura e quadril, massa gorda relativa e } \\
\text { absoluta, massa magra, flexibilidade, } \\
\text { força/resistência abdominal e aptidão } \\
\text { cardiorrespiratória, porém com resultados mais } \\
\text { expressivos para as meninas sobre a } \\
\text { circunferência de cintura, gordura corporal }(\mathrm{kg}) \text { e } \\
\text { força/resistência abdominal. }\end{array}$ \\
\hline Sousa et al., $2015^{29}$ & $\begin{array}{l}\text { - Uso de plataforma virtual (plataforma e-terapêutica } \\
\text { Next.Step) } \\
\text { - Apoio de equipe interdisciplinar (pediatra, nutricionista, } \\
\text { fisiologista e psicólogo) }\end{array}$ & $\begin{array}{l}\text { A avaliação pós-intervenção mostrou redução } \\
\text { significativa nas variáveis antropométricas e } \\
\text { comportamentais, z-score do IMC, percentil de } \\
\text { circunferência da cintura e do quadril e no tempo } \\
\text { de tela semanal. }\end{array}$ \\
\hline Fidelix et al., $2015^{30}$ & $\begin{array}{l}\text { - Consulta com endocrinologista } \\
\text { - Intervenção nutricional } \\
\text { - Psicoterapia } \\
\text { - Treinamento aeróbico supervisionado }\end{array}$ & $\begin{array}{l}\text { Adolescentes mais velhos, com maiores sintomas } \\
\text { de distúrbios alimentares e de níveis de colesterol } \\
\text { total, têm menor chance de aderir a tratamentos } \\
\text { interdisciplinares para perda de peso. }\end{array}$ \\
\hline $\begin{array}{l}\text { Hintze;Cattai;Junior, } \\
\qquad 2012^{36}\end{array}$ & $\begin{array}{l}\text { - Atividades físicas e treinos de resistência } \\
\text { - Intervenções nutricionais }\end{array}$ & $\begin{array}{l}\text { O programa resultou em melhorias na mudança } \\
\text { de comportamento no consumo de lipídios na } \\
\text { dieta e frequência de exercícios e na } \\
\text { autodescrição de atividade física habitual, } \\
\text { gordura corporal e aparência. }\end{array}$ \\
\hline $\begin{array}{l}\text { Denielsson et } \\
\text { al.,2012 }\end{array}$ & $\begin{array}{l}\text { - Acompanhamento periódico com orientações sobre } \\
\text { hábitos alimentares mais saudáveis e prática de } \\
\text { atividade física }\end{array}$ & $\begin{array}{l}\text { Os adolescentes entre } 14 \text { e } 16 \text { anos de idade não } \\
\text { apresentaram redução no escore do IMC após } 1 \text {, } \\
2 \text { ou } 3 \text { anos de tratamento comportamental. Por } \\
\text { outro lado, o efeito do tratamento } \\
\text { comportamental, quando iniciado entre } 6 \text { e } 9 \text { anos }\end{array}$ \\
\hline
\end{tabular}




\section{Revista Electrónica Enfermeria Actual en costa Rica}

\begin{tabular}{|c|}
\hline Silva et al.,2012 \\
\hline \\
\hline Martelo, 2009 \\
\hline Ishak et al., $2020^{15}$ \\
\hline Poll et al., 2019 \\
\hline Parra-Medina et \\
al.,2015 \\
\hline al., 2018 \\
\hline Chew et al.,2019 \\
\hline Rranco et al., 2019 \\
\hline Ryder et al.,2014 \\
\hline
\end{tabular}

- Terapia psicológica

- Orientação nutricional

- Atividade física orientada

- Consultas médicas individuais

- Exercício aeróbio, anaeróbio e intervenção nutricional

- Exercício aeróbio e intervenção nutricional

- Alimentação saudável

- Imagem corporal positiva

- Prática de atividade física

- Prática de atividade física

- Encontro semanal com psicólogo

- Atividades semanais sobre nutrição

- Orientações sobre aspectos nutricionais e reeducação alimentar

- Encontro semanal com psicólogo

- Controle de Ansiedade e Sentimentos Negativos /

Automonitoramento

- Aulas de educação nutricional

- Sessões de exercícios em grupo

- Atividades de integração sobre adoção de hábitos saudáveis de alimentação e à prática de atividade física - Jogos e campeonatos

- Atendimento individual sobre comportamento alimentar

- Dinâmicas em grupo

- Encontros grupais com pais e filhos

- Adoção de escolhas alimentares saudáveis

- Prática de atividade física

- Reeducação alimentar

-Prática de atividades físicas (200 minutos por semana)

- Uma sessão individual de 30 minutos com a

nutricionista

- Sessões de educação em grupo duas vezes por semana

- Prática de atividades físicas três vezes por semana de idade foi mais eficaz com obesidade grave do que com obesidade moderada.

As intervenções promovem melhorias nos parâmetros antropométricos, IMC, circunferência da cintura e massa gorda absoluta. Além de melhor composição corporal e aptidão física

O grupo com exercício aeróbio, anaeróbio e intervenção nutricional obteve melhores resultados, com diminuição do IMC, prega cutânea subescapular, gordura corporal, e, aumento da massa magra.

Houve efeito positivo no conhecimento dos adolescentes sobre estilo de vida saudável, mas sem redução significativa da composição corporal.

Houve redução do IMC e circunferência da cintura, diminuição de alimentos ultraprocessados, tendência de melhora da qualidade de vida, contudo, houve redução dos domínios da qualidade de vida emocional e escolar.

Os resultados mostraram reduções na massa gorda, gordura corporal e circunferência da cintura, bem como, aumentos na massa musculoesquelética e na taxa metabólica de repouso. No entanto, não houve diferenças significativas na massa corporal, IMC, circunferência do pescoço ou circunferência do braço.

A intervenção resultou em concentrações mais baixas de LDL $(\mathrm{p}=0,001)$ e Selectina $(\mathrm{p}=0,008)$ nos adolescentes obesos.

Houve redução na frequência de consumo de lanches fast food na escola, intervenção comparada ao controle $(\mathrm{p}=0,001)$.

A intervenção foi eficaz para ajudar os adolescentes a compreenderem sua história de vida e determinantes do comportamento alimentar, assim como efetivarem mudanças na sua alimentação espontaneamente.

O programa mostrou viabilidade e eficácia clínica a curto prazo em melhorar alguns resultados clínicos e a percepção dos adolescentes sobre o apoio familiar.

A intervenção intensiva no estilo de vida reduziu o IMC em crianças com obesidade abdominal. Houve melhora significativa de índices alimentares, aproximando-se das recomendações nutricionais.

O IMC, peso, circunferência da cintura e percentual de gordura corporal melhoraram 


\section{Revista Electrónica Enfermeria Actual en costa Rica}

\begin{tabular}{|c|}
\hline Sousa et al., 2014 \\
\hline Giel et al.,2013 \\
\hline Martín-García et al., \\
2019 \\
\hline Rubin et al., 2019² \\
\hline Duman et al., 2016 \\
\hline Marker-Hoffman, \\
2012 \\
\hline Milano et al., 2013 \\
\hline Antunes et al., 2013 \\
\hline $2016^{27}$ \\
\hline Tortosa-Martínez et \\
al., 2016
\end{tabular}

- Alimentação saudável e educação para a atividade física - Mudanças ambientais e organizacionais no ambiente escolar

- Aumento da atividade física de 1 para 2 horas por dia - Diminuição de 2 horas na televisão e computador - Orientações nutricionais

- 90 minutos de jogos recreativos duas vezes por semana - Estratégia motivacional com feedback constante

- Jogo ativo em casa com equipamentos (bolas, argolas, obstáculos e cones)

- Ligações aos pais (orientação do jogo/ aconselhamento/solução de problemas)

- Programa de exercícios de oito semanas por três dias por semana (exercícios de aquecimento, programa de exercícios e relaxamento)

- Videogames ativos e exercícios aeróbicos e calistênicos acompanhados de música

- Sessões de aquecimento (5 a 10 minutos)

- Atividade física moderada a vigorosa (65-70 minutos)

- Alongamentos, exercícios respiratórios e a técnica de relaxamento (10 minutos)

- Prática de atividades físicas três vezes por semana e duração de 60 minutos

- Intervenção nutricional e psicológica quinzenalmente

- Treinamento concorrente com 30 minutos de atividades aeróbicas (caminhada ou corrida) e 30 minutos de trabalho resistido com exercícios com pesos

Atividades aeróbicas três vezes por semana, com sessões de 110 minutos

- Jogo de realidade alternativa significativamente entre adultos que completaram o programa, mas as crianças e adolescentes participantes não apresentaram melhorias.

Houve estabilização da prevalência de obesidade geral e central no GI. Verificou-se o aumento da prevalência para o grupo controle, para IMC e razão cintura-estatura.

Os participantes mostraram uma mudança significativa de peso, com diminuição média de $0,1 \pm 0,2$ IMC $(p<0,01)$. No entanto, a prevalência de sintomas de transtorno alimentar permaneceu estável após as intervenções.

A intervenção influenciou os comportamentos alimentares dos jovens, principalmente o fator alimentação emocional e promoveu mudanças favoráveis na composição corporal.

Esta intervenção guiada pelos pais não aumentou a atividade física. No entanto, levou a melhorias na competência bruta das habilidades motoras.

Exercícios aplicados com videogames ativos têm efeitos positivos no estado obeso, status psicossocial e autoestima, promovendo melhoria do estado obeso, além de ter contribuições positivas para o bem-estar psicológico.

O programa de atividade físico-esportiva contribuiu com a melhoria de parâmetros cardiovasculares e na composição corporal.

O programa de exercícios associado com aconselhamento nutricional e psicológico foi capaz de promover benefícios na modulação autonômica, reduzir a frequência cardíaca de repouso e mudar a classificação da obesidade dos indivíduos.

O treinamento concorrente foi eficaz para promover a redução da prevalência de fígado gorduroso, diminuição do tamanho dos lóbulos hepáticos e alterações nos parâmetros de composição corporal.

Após o tratamento com exercício físico, os valores da atividade da enzima butirilcolinesterase dos adolescentes obesos tornaram-se semelhantes àqueles verificados em indivíduos eutróficos.

Houve associação significativa para aumento da atividade física no grupo submetido ao jogo de realidade alternativa $(\mathrm{p}<0,001)$. Ao contrário do grupo comparação, em que houve diminuição significativa $(\mathrm{p}=0,001)$. 


\section{Revista Electrónica Enfermeria Actual en costa Rica}

\begin{tabular}{|c|l|}
\hline Farias et al., 200942 & $\begin{array}{l}\text { - Aulas de educação física semanais, com duração de } 60 \\
\text { minutos }\end{array}$ \\
\hline $\begin{array}{c}\text { Fernandez et al., } \\
\mathbf{2 0 0 4 4}\end{array}$ & $\begin{array}{l}\text { - Treinamento físico aeróbico e anaeróbico } \\
\text { - Orientação nutricional }\end{array}$ \\
\hline Freitas et al., 201733 & $\begin{array}{l}\text { - Aconselhamento semanal por uma hora por psicólogo } \\
\text { clínico (imagem corporal, distúrbios alimentares, relação } \\
\text { entre alimentos e sensações, problemas familiares e } \\
\text { sociais, humor, ansiedade e depressão) }\end{array}$ \\
\hline
\end{tabular}

Fonte: Elaborado pelos autores.
A atividade física realizada durante a atividade escolar promoveu redução significativa da gordura corporal.

O exercício anaeróbio, proposto neste estudo, foi mais eficiente para promover a diminuição da gordura corporal e da percentagem de gordura que o exercício aeróbio e a orientação alimentar isolada.

Os adolescentes que receberam a intervenção apresentam menor peso corporal, massa gorda relativa, maior massa livre de gordura e melhora na qualidade de vida.

\section{DISCUSSÃO}

Esta revisão incluiu 31 estudos que abrangeram programas de intervenção com foco na redução de peso de adolescentes. Os cenários predominantes foram os espaços familiares e escolares, por constituírem-se como ambientes de fácil acesso aos adolescentes. Deles, 29 estudos apresentaram resultados significativos nos parâmetros avaliados após as intervenções.

Os estudos analisados buscaram a inclusão de adolescentes com IMC elevado (>19). A determinação do IMC tem sido adotada como método simples de avaliação do estado nutricional de diferentes grupos populacionais, inclusive adolescentes ${ }^{16,18,19}$. Na América Latina, a prevalência do excesso de peso oscila de 19 a $37 \%$ na faixa etária de 5 a 11 anos e de 17 a $36 \%$ na faixa etária de 12 e $19 \operatorname{anos}^{43}$.

Estudos têm demonstrado que isso se deve ao fato de adolescentes possuírem preferências alimentares inadequadas, com alto consumo de alimentos processados e ultraprocessados, por interferência das relações sociais existentes e midiáticas, o que favorece elevação do peso e, consequentemente, do IMC ${ }^{44,45}$. Além disso, adiciona-se a ocorrência do uso generalizado da tecnologia inclusive em atividades de lazer como televisão, videogames, computadores, tablets e celulares, que impulsionam o comportamento sedentário ${ }^{46}$, demonstrando a necessidade de intervenções nesta condição crônica ${ }^{18}$.

Para contribuir com a redução da prevalência do excesso de peso entre adolescentes, estudos clínicos têm buscado desenvolver programas de intervenção educativa longitudinais que possam favorecer a mudança no estilo de vida, reeducação alimentar, perda de peso e melhor qualidade de vida ${ }^{6,22}$.

No presente estudo, foram observadas intervenções que abrangeram orientações pautadas no apoio nutricional e psicológico, prática de atividade física ou exercício físico e estilo de vida saudável. Tais estratégias correspondem aos principais tipos de tratamentos não farmacológicos da obesidade e costumam ter influência significativa na saúde do indivíduo ${ }^{18}$.

No que se refere aos programas de intervenção pautados em exercícios físicos é importante ressaltar sua relevância na redução da obesidade em adolescentes, devido ao aumento da taxa metabólica de repouso e massa livre de gordura, que consequentemente aumentam o gasto energético diário ${ }^{47}$. Estudo consultado que avaliou os efeitos de 


\section{Revista Electrónica Enfermeria Actual en costa Rica}

programas de exercícios físicos na composição corporal, aptidão física e risco cardiometabólico em adolescentes identificou impacto positivo na composição corporal e nas variáveis antropométricas ${ }^{19}$.

A prática de 60 minutos de atividade física por dia, permeada por movimentos que aumentem o consumo de energia, está associada a resultados fisiológicos e psicológicos, que podem propiciar melhora no perfil da composição corporal $^{48}$. Exercícios físicos orientados podem ser importante estratégia na redução de medidas antropométricas e no desenvolvimento físico do adolescente, porém, é válido destacar a necessidade de associar exercícios com mudanças nos hábitos alimentares e apoio familiar para redução efetiva na obesidade e manutenção dos resultados obtidos.

Outra intervenção de destaque foram os programas comportamentais com elementos de intervenção interdisciplinares, uma vez que favoreceram a redução do peso corporal, promoção de práticas saudáveis e mudanças nos comportamentos relativos ao estilo de vida ${ }^{28}$.

Estudos consultados reportam a relevância desse método para redução de peso de adolescentes com IMC elevados ${ }^{7,8}$. Intervenções com elementos interdisciplinares, em associação com educação física, enfermagem, fisioterapia, nutrição e psicologia podem impactar positivamente na redução de circunferências, massa gorda e percentual de gordura corporal de adolescentes, além da melhora na imagem corpora ${ }^{17,49}$. Esses achados ressaltam a importância no cuidado interdisciplinar no combate à obesidade infanto-juvenil e no envolvimento dos profissionais em ações de promoção da saúde destinadas a essa população.

Logo, intervenções interdisciplinares com adolescentes podem favorecer a aquisição de melhores hábitos alimentares e serem mantidos durante toda a vida adulta $^{50}$. Além disso, foi possível associar as intervenções comportamentais com outras estratégias, sejam educativas, sociais e ambientais, garantindo melhores resultados a longo prazo.

A educação nutricional e alimentar também foi utilizada para reduzir o IMC e outros aspectos de saúde de adolescentes. Essa intervenção é considerada estratégia de tratamento não farmacológico de baixo custo para promoção da saúde em adolescentes com sobrepeso e obesidade, uma vez que, a medida que o estado nutricional adequado é restabelecido, as médias do IMC o acompanharão ${ }^{49,51}$.

Programa de intervenção que mantém os adolescentes mais próximos com nutricionista e equipe de saúde pode propiciar maior eficácia na perda de peso. Contudo, os adolescentes precisam de tempo para mudar seus hábitos alimentares e exigem esforço contínuo para manter um novo estilo de vida ${ }^{52}$. Isso reforça a relevância de programas de intervenção de cunho longitudinal para que as ações gerem resultados positivos e duradouros, além de beneficiarem alcance da perda ponderal e melhor estado de saúde.

Alguns programas focaram em intervenção no estilo de vida, em que a maioria associou estratégias de mudanças comportamentais que pudessem favorecer a adoção de práticas alimentares saudáveis e atividade física. Intervenções de estilo de vida saudável em vários níveis com adolescentes, principalmente quando envolvem a família, são abordagens promissoras para melhorar os comportamentos individuais ${ }^{26}$.

Pesquisa na Espanha identificou que intervenção intensiva no estilo de vida em crianças e adolescentes com obesidade abdominal alcançou redução no IMC, melhor adesão às recomendações nutricionais e escores mais altos 


\section{Revista Electrónica Enfermeria Actual en costa Rica}

de qualidade da dieta ${ }^{22}$. Esse dado infere na importância da manutenção do estilo de vida saudável para alcance de resultados favoráveis relacionados ao peso corporal.

Dentro dos cuidados de saúde com a obesidade em adolescentes, destaca-se que a inclusão de um componente de aconselhamento psicológico na prevenção e tratamento multidisciplinar para obesidade pode fornecer melhores benefícios ${ }^{6}$.

Os estudos analisados na revisão sugerem efetividade dos programas de intervenção educativa em promover mudanças positivas no conhecimento dos adolescentes sobre estilo de vida saudável ${ }^{16}$, redução do IMC $^{8,18,19,22,26}$, diminuição no consumo de alimentos ultraprocessados ${ }^{18}$, melhora na imagem corpora ${ }^{17}$ e melhor qualidade de $\operatorname{vida}^{6,18}$.

Ao interpretar tais resultados também foi preciso levar em consideração que diversos fatores podem influenciar a efetividade de programas de intervenção com foco na obesidade, como condição social, acesso aos serviços de saúde, aspectos culturais e nível de instrução educacional, além da participação interdisciplinar nessas atividades.

Apesar da inclusão de estudos primários com altos níveis de evidência, observaram-se limitações na padronização dos desfechos e na redação dos estudos, fato que limitou a coleta de informações importantes.

Outra limitação potencial do estudo é o viés de publicação, uma vez que os achados positivos têm maior probabilidade de serem publicados, resultando em uma valorização do efeito benéfico das intervenções no controle ou tratamento da obesidade. Destaca-se ainda o fato da busca ter ocorrido com os descritores no idioma inglês, de forma que os artigos sem a disponibilização no referido idioma e que seriam elegíveis para integrar a amostra do estudo podem não ter sido encontrados.

Novos estudos sobre a temática são esperados, a fim de produzir novas evidências sobre mudanças no comportamento de adolescentes com sobrepeso e obesidade de modo que se tenham mais controle sobre os benefícios e a adesão a intervenções que objetivam prevenir e tratar a obesidade.

\section{CONCLUSÃO}

As intervenções educativas analisadas demonstraram efetividade das intervenções educativas no conhecimento dos adolescentes e estilo de vida saudável, que contribuiu com redução do IMC e outros parâmetros corporais, como gordura corporal e circunferência abdominal, menor ingestão de alimentos ultraprocessados, melhora na imagem corporal e qualidade de vida.

\section{Conflito de interesse}

Os autores declaram que não possuem conflito de interesse de qualquer natureza relacionado ao artigo.

\section{REFERENCIAS}

1. World Health Organization (WHO). Obesity and overweight. Genève: WHO; 2020. Disponível em: https://www.who.int/news-room/fact-sheets/detail/obesity-and-overweight 


\section{Revista Electrónica Enfermeria Actual en costa Rica}

2. Ferreira APS, Szwarcwald CL, Damacena GN. Prevalence of obesity and associated factors in the Brazilian population: a study of data from the 2013 National Health Survey. Rev. Bras Epidemiol. 2019; 22: e190024. DOI: http://dx.doi.org/10.1590/1980-549720190024

3. Sanyaolu A, Okorie C, Qi X, Locke J, Rehman S. Childhood and Adolescent Obesity in the United States: A Public Health Concern. Glob Pediatr Health. 2019; 6(1): 1-11. DOI: http://dx.doi.org/10.1177/2333794X19891305

4. Abduelkarem AR, Sharif SI, Bankessli FG, Kamal SA, Kulhasan NM, Hamrouni AM. Obesity and its associated risk factors among school-aged children in Sharjah, UAE. PLoS ONE. 2020; 15(6): e0234244. DOI: https://doi.org/10.1371/journal. pone.0234244

5. Spinelli A, Buoncristiano M, Kovacs VA, Yngve A, Spiroski I, Obreja G, et al. Prevalence of Severe Obesity among Primary School Children in 21 European Countries. Obes Facts. 2019; 12(2):244-258. DOI: https://doi.org/10.1159/000500436

6. Freitas CRM, Gunnarsdottir T, Fidelix YL, Tenório TRS, Lofrano-Prado MC, Hill JO et al. Effects of a psychological intervention on the quality of life of obese adolescents under a multidisciplinary treatment. J. Pediatr. 2017; 93 (2): 185-191. DOI: http://dx.doi.org/10.1016/j.jped.2016.05.009

7. Costa L, Rubio KL, Lopes SMA, Tanouye ATA, Bertolini SMMG, Branco BHM. Effects of 12 weeks of interdisciplinary interventions on behavioral and eating parameters of adolescents with over weight or obesity. J. Hum. Growth Dev. 2019; 29 (2): 177-186. DOI: http://doi.org/10.7322/jhgd.v29.9417

8. Bianchini JAA, Silva DFDA, Lopera CA, Antonini VDS, Nardo Junior N. Intervenção multiprofissional melhora a aptidão física relacionada à saúde de adolescentes com maior efeito sobre as meninas em comparação aos meninos. Rev. Bras. Educ. Fís. Esporte. 2016; 30 (4): 1051-1059. DOI: https://doi.org/10.1590/1807-55092016000401051

9. Lima TR, Moraes MS, Andrade JHC, Farias JM, Silva Diego AS. Fatores associados à presença isolada e simultânea de excesso de peso e obesidade abdominal em adolescentes. Rev. Paul. Pediatr. 2020; 38: e2018332. DOI: https://doi.org/10.1590/1984-0462/2020/38/2018332

10. Braga VAS, Jesus MCP, Conz CA, Silva MH, Tavares RE, Merighi MAB. Actions of nurses toward obesity in primary health careunits. Rev. Bras. Enferm. 2020; 73 (2): e20180404. DOI: http://dx.doi.org/10.1590/0034-7167-2018-0404

11. Ramalho NJM, Marques DKA, Fernandes MGM, Nóbrega MML. Análise de teorias de enfermagem de Meleis: revisão integrativa. Rev. Bras. Enferm. 2016; 69 (1): 174-181. DOI: http://dx.doi.org/10.1590/0034-7167.2016690123i

12. Mendes KDS, Silveira RCCP, Galvão CM. Revisão integrativa: método de pesquisa para a incorporação de evidências na saúde e na enfermagem. Texto \& Contexto: Enferm. 2008; 17 (4): 758-764. DOI: https://doi.org/10.1590/S0104-07072008000400018

13. Lockwood C, Porrit K, Munn Z, Rittenmeyer L, Salmond S, Bjerrum M, et al. Chapter 2: Systematic reviews of qualitative evidence. In: Aromataris E, Munn Z, editors. Joanna Briggs Institute, 2017. Disponível em: https://reviewersmanual.joannabriggs.org

14. Melnyk BM, Fineout-Overholt H. Evidence-based practice in nursing and health care: a guide to Best practice. Philadelphia: Lippincott Williams \& Wilkins; 2005.

15. Zanatta LB, Heinzmann-Filho JP, Vendrusculo FM, Campos NE, Oliveira MS, Feoli AM, et al. Effect of na interdisciplinary intervention with motivational approach on exercise capacity in obese adolescents: a randomized controlled clinical trial. Einstein. 2020; 18: eAO5268. DOI: http://dx.doi.org/10.31744/einstein_journal/2020AO5268 


\section{Revista Electrónica Enfermeria Actual en costa Rica}

16. Sharif Ishak SIZ, Chin YS, MohdTaib MN, Chan YM, MohdShariff Z. Effectiveness of a school-based intervention on knowledge, attitude and practice on health y lifestyle and body composition in Malaysian adolescents. BMC Pediatr. 2020; 14; 20 (1): 122. DOI: http://dx.doi.org/10.1186/s12887-020-02023-X

17. Chew CSE, Oh JY, Rajasegaran K, Saffari SE, Lim CMM, Lim SC, et al. Evaluation of a group familybased intervention programme for adolescent obesity: the LITE randomised controlled pilot trial. Singapore Med. J. 2019; 10.11622/smedj.2019122. DOI: https://doi.org/10.11622/smedj.2019122

18. Poll FA, Miraglia F, D'avila HF, Reuter CP, Mello ED. Impact of intervention on nutritional status, consumption of processed foods, and quality of life of adolescents with excess weight. J. Pediatr. 2019; S0021-7557(19)30152-4. DOI: https://doi.org/10.1016/j.jped.2019.05.007

19. Branco BHM, Valladares D, Oliveira FM, Carvalho IZ, Marques DC, Coelho AA, Oliveira LP and Bertolini SMMG. Effects of the order of physical exerciseson body composition, physical fitness, and cardiometabolic risk in adolescents participating in na interdisciplinary program focusingon the treatment of Obesity. Front Physiol. 2019; 10: 1013. DOI: https://doi.org/10.3389/fphys.2019.01013

20. Martín-García M, Alegre LM, García-Cuartero B, Bryant EJ, Gutin B, Ara I. Effects of a 3-month vigorous physical activity intervention on eating behaviors and body composition in over weight and obese boys and girls. J. Sport Health Sci. 2019; 8 (2): 170-6. DOI: https://doi.org/10.1016/j.jshs.2017.09.012

21. Rubin DA, Wilson KS, Dumont-Driscoll M, Rose DJ. Effectiveness of a parent-led physical activity intervention in youth with obesity. Med. Sci. Sports Exerc. 2019; 51 (4): 805-813. DOI: https://doi.org/10.1249/MSS.0000000000001835

22. Ojeda-Rodríguez A, Zazpe I, Morell-Azanza L, Chueca MJ, Azcona-Sanjulian MC, Marti A. Improved diet quality and nutrient adequacy in children and adolescents with abdominal obesity after a lifestyle intervention. Nutrients. 2018; 10 (10): 1500. DOI: http://doi.org/10.3390/nu10101500

23. Duman F, Kokaçya MH, Doğru E, Katayıfcı N, Canbay Ö, Aman F. The Role of active video: accompanied exercises in improvement of the obese state in children: a prospective study from Turkey. J. Clin. Res. Pediatr. Endocrinol. 2016; 8 (3): 334-340. DOI: https://doi.org/10.4274/jcrpe.2284

24. Tortosa-Martínez M, Gil-Madrona P, Pastor-Vicedo JC, Contreras-Jordán O. programa de actividad física extracurricular en adolescentes con sobrepeso u obesidad. Rev. Latinoam. Cienc. Soc. Niñez. 2016; 14 (1): 577-589. DOI: https://doi.org/10.11600/1692715x.14139190315

25. Ricci-Vitor AL, Rossi FE, Hirai PM, da Silva NT, Vanderlei FM, Haddad MI et al. Effects of a multidisciplinary program on autonomic modulation in over weight or obese children and adolescents. J. Hum. Growth Dev. 2016; 26 (2), 154-161. DOI: https://dx.doi.org/10.7322/jhgd.119257

26. Parra-Medina D, Liang Y, Yin Z, Esparza L, Lopez L. Weight out comes of latino adults and children participating in the Y Living Program, a family-focused lifestyle Intervention, San Antonio, 2012-2013. Prev. Chronic Dis. 2015; 12: 150219. DOI: http://dx.doi.org/10.5888/pcd12.150219

27. Souza FJR, Vasconcelos EO, Lopes JPN, Letieri RV, Pontes Junior JAF. Comportamento ativo, sedentário e habitos alimentares de adolescentes de uma cidade do interior do Ceará, Brasil. Conexões. 2015; 13 (1): 114-28. DOI: https://doi.org/10.20396/conex.v13i1.2152

28. Fidelix YL, Farias Júnior JC, Lofrano-Prado MC, Guerra RLF, Cardel M, Prado WL. Multidisciplinary intervention in obese adolescents: predictors of drop out. Einstein. 2015; 13 (3): 388-394. DOI: https://doi.org/10.1590/S1679-45082015AO3339 


\section{Revista Electrónica Enfermeria Actual en costa Rica}

29. Ryder E, Diez-Ewald M, Mosquera J, Fernández E, Pedreañez A, Vargas R et al. Association of obesity with leukocyte count in obese individuals with out metabolic syndrome. Diabetes Metab. Syndr. 2014; 8 (4): 197-204. DOI: https://doi.org/10.1016/j.dsx.2014.09.002

30. Souza MCC, Tibúrcio JD, Bicalho JMF, Rennó HMS, Dutra JS, Campos LG et al. Factors associated with obesity and over weight in school-aged children. Texto \& Contexto: Enfermagem. 2014; 23(3): 712-719. DOI: https://doi.org/10.1590/0104-07072014001740013

31. Giel KE, Zipfel S, Schweizer R, Braun R, Ranke MB, Binder G et al. Eating disorder pathology in adolescents participating in a life style intervention for obesity: association swith weight change, general psychopathology and health-related quality of life. Obes. Facts. 2013; 6 (4): 307-316. DOI: https://doi.org/10.1159/000354534

32. Antunes Bde M, Monteiro PA, Silveira LS, Cayres SU, Silva CB, F IF Jr. Effect of concurrent training on risk factors and hepatic steatosis in obese adolescents. Rev Paul Pediatr. 2013; 31 (3): 371-6. DOI: https://doi.org/10.1590/S0103-05822013000300015

33. Milano GE, Leite N, Chaves TJ, Milano GE, Souza RLR, Alle LF. Atividade da butirilcolinesterase e fatores de risco cardiovascular em adolescentes obesos submetidos a um programa de exercícios físicos. Arq. Bras. Endocrinol Metab. 2013; 57 (7): 533-537. DOI: https://doi.org/10.1590/S000427302013000700006

34. Hintze LJ, Cattai GBP, Nardo Junior N. Multidisciplinary program for obesity treatment: summary of results with adolescentes. Acta Sci. Health Sci. 2012; 34 (2): 137-44. DOI: https://doi.org/10.4025/actascihealthsci.v34i2.8934

35. Danielsson P, Kowalski J, Ekblom Ö, Marcus C. Response of severely obese children and adolescents to behavioral treatment. Arch. Pediatr. Adolesc. Med. 2012; 166 (12): 1103-1108. DOI: https://doi.org/10.1001/2013.jamapediatrics.319

36. Silva DF, Souza LL, Delfino RO, Bianchini JAA, Hintze LJ, Nardo Junior N. Efeitos de um programa multiprofissional de tratamento da obesidade e de sua cessação sobre a aptidão física relacionada à saúde de adolescentes. Rev. Educ. Fis UEM. 2012; 23 (3): 399-410. DOI: https://doi.org/10.4025/reveducfis.v23i3.14855

37. Johnston JD, Massey AP, Marker-Hoffman RL. Using na alternate reality game to increase physical activity and decrease obesity risk of college students. J. Diabetes Sci. Technol. 2012; 6 (4): 828-838. DOI: https://doi.org/10.1177/193229681200600414

38. Vargas ICS, Sichieri R, Sandre-Pereira G, Veiga GV. Avaliação de programa de prevenção de obesidade em adolescentes de escolas públicas. Rev. Saúde Pública. 2011; 45 (1): 59-68. DOI: https://doi.org/10.1590/S0034-89102011000100007.

39. Martelo S. Effects of nutritional education associated with exercise on body composition and biochemical parameters in adolescents with excess weight. Nutrire: Rev. Soc. Bras. Alim. Nutr. 2009; 34 (3): 31-44.

40. Farias ES, Paula F, Carvalho WR, Gonçalves EM, Baldin AD, Guerra-Júnior G. Influence of programmed physical activity on body composition among adolescent students. J. Pediatr. (Rio J). 2009; 85 (1): 28-34. DOI: https://doi.org/10.2223/JPED. 1864

41. Rodrigues EM, Boog MCF. Problematização como estratégia de educação nutricional com adolescentes obesos. Cad. Saúde Pública. 2006; 22 (5): 923-931. DOI: https://doi.org/10.1590/S0102$\underline{311 X 2006000500005}$ 


\section{Revista Electrónica Enfermeria Actual en costa Rica}

42. Fernandez AC, Mello MT, Tufik S, Castro PM, Fisberg M. Influência do treinamento aeróbio e anaeróbio na massa de gordura corporal de adolescentes obesos. Rev Bras Med Esporte. 2004; 10 (3): 1-7. Disponível em: https://www.scielo.br/pdf/rbme/v10n3/21143.pdf

43. Rivera JA, de Cossío TG, Pedraza LS, Aburto TC, Sánchez TG, Martorell R. Childhood and adolescent over weight and obesity in Latin America: a systematic review. Lancet Diabetes Endocrinol. 2014; 2(4): 321-32. DOI: https://doi.org/10.1016/S2213-8587(13)70173-6

44. Cardoso S, Santos O, Nunes C, Loureiro I. Escolhas e hábitos alimentares em adolescentes: associação com padrões alimentares do agregado familiar. Rev. Port. Saúde Pública. 2015; 33 (2): 128-136. DOI: https://doi.org/10.1016/j.rpsp.2014.07.004

45. Corrêa RS, Vencato PH, Rockett FC, Bosa VL. Padrões alimentares de escolares: existem diferenças entre crianças e adolescentes? Ciênc. Saúde Coletiva. 2017; 22 (2): 553-562. DOI: https://doi.org/10.1590/1413-81232017222.09422016

46. Popkin BM. Nutrition transition and the global diabetes epidemic. Curr. Diab. Rep. $2015 ; 15$ (9): 64. DOI: https://doi.org/10.1007/s11892-015-0631-4

47. Petridou A, Siopi A, Mougios V. Exercise in the management of obesity. Metabolism. 2019; 92: 163 169. DOI: https://doi.org/10.1016/j.metabol.2018.10.009

48. Hollis JL, Sutherland R, Williams AJ, Campbell E, Nathan N, Wolfenden L et al. A systematic review and meta-analysis of moderate-to-vigorous physical activity levels in secondary school physical education lessons. Int. J. Behav. Nutr. Phys. Act. 2017; 14, 52. DOI: https://doi.org/10.1186/s12966017-0504-0

49. Magnani Branco BH, Carvalho IZ, Garcia de Oliveira H, et al. Effects of 2 types of resistance training models on obese adolescents' body composition, cardiometabolic risk, and physical fitness. J. Strength Cond. Res. 2020; 34 (9): 2672-2682. DOI: https://doi.org/10.1519/JSC.0000000000002877

50. Florido LMP, Mulaski LFS, Hespanhol MB, Mendonça RCS, Santos TC, Silva WAS. Fightingagainstobesity: behavioral and alimentarystrategies. Revista Caderno de Medicina. 2019; 2 (2): 80-89.

51. Sousa JCS, Oliveira CM, Araújo ASM, Santo RD, Fernandes LL, Serquiz AC. Avaliação do estado nutricional de pacientes hospitalizados: uso de métodos convencionais e não convencionais. Demetra. 2018; 13 (1): 195-208. DOI: https://doi.org/10.12957/demetra.2018.29094

52. Gibson AA, Sainsbury A. Strategies to Improve Adherence to Dietary Weight Loss Interventions in Research and Real-World Settings. Behav Sci (Basel). 2017; 7(3):44. DOI: https://doi.org/10.3390/bs7030044 